Pacific

Journal of

Mathematics

THE HARMONICITY OF THE REEB VECTOR FIELD ON CONTACT METRIC 3-MANIFOLDS

Themis Koufogiorgos, Michael Markellos

AND VASSILIS J. PAPANTONIOU 


\title{
THE HARMONICITY OF THE REEB VECTOR FIELD ON CONTACT METRIC 3-MANIFOLDS
}

\author{
Themis Koufogiorgos, Michael Markellos \\ AND VASSILIS J. PAPANTONIOU
}

\begin{abstract}
A contact metric manifold whose characteristic vector field is a harmonic vector field is called an $\boldsymbol{H}$-contact metric manifold. We introduce the notion of $(\kappa, \mu, v)$-contact metric manifolds in terms of a specific curvature condition. Then, we prove that a contact metric 3-manifold $M$ is an $H$-contact metric manifold if and only if it is a $(\kappa, \mu, v)$-contact metric manifold on an everywhere open and dense subset of $M$. Also, we prove that, for dimensions greater than three, such manifolds are reduced to $(\kappa, \mu)$-contact metric manifolds whereas, in three dimensions, $(\kappa, \mu, v)$-contact metric manifolds exist.
\end{abstract}

\section{Introduction}

Let $(M, g)$ be a Riemannian manifold and $\left(T_{1} M, g_{S}\right)$ its unit tangent sphere bundle equipped with the Sasaki metric $g_{S}$. If $M$ is compact and orientable, then a unit vector field $V$ on $M$ is called harmonic if it is a critical point for the energy functional restricted to the space $\mathscr{L}^{1}(M)$ of all unit vector fields on $(M, g)$. The corresponding critical point condition has been determined in [Wiegmink 1995] and [Wood 1997]. On the other hand, a harmonic vector field determines a harmonic map $\left((M, g) \mapsto\left(T_{1} M, g_{S}\right)\right)$ if an additional condition is satisfied; see Section 2. The first examples of harmonic vector fields were the Hopf vector fields on the unit odd-dimensional spheres, that is, the Reeb vector fields of the standard Sasakian structure of the unit odd dimensional spheres [Han and Yim 1998]. So, it is natural to study the harmonicity of the Reeb vector field of a general contact Riemannian manifold and to explore how its harmonicity is related to the geometry of the manifold.

A $(2 n+1)$-dimensional contact metric manifold $M(\eta, \xi, \phi, g)$ whose characteristic vector field $\xi$ is a harmonic vector field is called an $H$-contact metric manifold.

MSC2000: primary 53D10; secondary 53C25, 53C15.

Keywords: contact metric manifolds, harmonic characteristic vector fields, H-contact manifolds,

$(\kappa, \mu, v)$-contact metric manifolds.

Markellos was partially supported by the Greek State Scholarships Foundation (I.K.Y.) and by the C. Carathéodory grant no.C.161 2007-10, University of Patras. 
D. Perrone [2004] proved that $M(\eta, \xi, \phi, g)$ is $H$-contact metric manifold if and only if $\xi$ is an eigenvector of the Ricci operator, generalizing the same result of J. C. González- Dávila and L. Vanhecke [2001] for $n=1$. It is important to mention that the class of $H$-contact metric manifolds includes several interesting classes of contact metric manifolds such as Sasakian and $\eta$-Einstein manifolds, $K$-contact manifolds, strongly $\phi$-symmetric spaces, $(\kappa, \mu)$-contact metric manifolds, and generalized $(\kappa, \mu)$-contact metric manifolds. Perrone [2003] also gave a geometric interpretation of generalized $(\kappa, \mu)$-contact metric manifolds in terms of harmonic maps. In particular, he showed that a contact metric 3-manifold $M$ is a generalized $(\kappa, \mu)$-contact metric manifold on an everywhere dense open subset of $M$ if and only if its characteristic vector field $\xi$ determines a harmonic map.

The paper is organized as follows. In Section 2, we present some basic notions about contact manifolds and harmonic vector fields. Also, we introduce the notion of $(\kappa, \mu, v)$-contact metric manifolds.

In Section 3, we characterize the 3-dimensional $H$-contact metric manifolds in terms of $(\kappa, \mu, v)$-contact metric manifolds. In particular, we prove this theorem:

Theorem 1.1. Let $M(\eta, \xi, \phi, g)$ be a 3-dimensional contact metric manifold. If $M$ is a $(\kappa, \mu, v)$-contact metric manifold, then $M$ is an $H$-contact metric manifold. Conversely, if $M$ is a 3-dimensional $H$-contact metric manifold, then $M$ is a $(\kappa, \mu, v)$-contact metric manifold on an everywhere open and dense subset of $M$.

In Section 4, we ask if there exist $(\kappa, \mu, v)$-contact metric manifolds such that $\kappa, \mu, v$ are smooth functions independent of the choice of the vector fields $X, Y$. We prove that the answer is affirmative for dimension greater than three but negative for dimension equal to three. The main result of Section 4 is this:

Theorem 1.2. Every $(\kappa, \mu, v)$-contact metric manifold $M(\eta, \xi, \phi, g)$ of dimension greater than 3 is either a Sasakian manifold or a $(\kappa, \mu)$-contact metric manifold, that is, the functions $\kappa, \mu$ are constants and $v$ is the zero function.

In a sense this result is optimal. In dimension 3, we construct a one-parameter family of 3-dimensional $(\kappa, \mu, v)$-contact metric manifolds using that these manifolds are invariant under $D$-homothetic transformations (see Section 3).

Finally, in Section 5 we attempt to classify all 3-dimensional $(\kappa, \mu, v)$-contact metric manifolds. We give partial answers to this problem under the assumption that these manifolds additionally satisfy some specific geometric conditions.

\section{Preliminaries}

2.1. Contact metric manifolds. Here, we present some basic facts about contact manifolds. All manifolds are assumed to be connected and smooth. A differentiable $(2 n+1)$-dimensional manifold is called contact manifold if it admits a global 
1-form $\eta$ such that $\eta \wedge(d \eta)^{n} \neq 0$ everywhere on $M$. It is known that a contact manifold admits an almost contact metric structure $(\eta, \xi, \phi, g)$, that is, it admits global vector field $\xi$, called the characteristic vector field or the Reeb vector field, a tensor field $\phi$ of type $(1,1)$, and a Riemannian metric $g$ (associated metric) such that

$$
\begin{aligned}
\eta(\xi) & =1, & \phi^{2} & =-I d+\eta \otimes \xi, \\
\eta \circ \phi & =0, & g(\phi X, \phi Y) & =g(X, Y)-\eta(X) \eta(Y)
\end{aligned}
$$

for all vector fields $\mathrm{X}, \mathrm{Y}$ on $M$. The quadruple $(\eta, \xi, \phi, g)$ can be chosen so that $d \eta(X, Y)=g(X, \phi Y)$. The manifold $M$ together with the structure tensors $(\eta, \xi, \phi, g)$ is called a contact metric manifold and is denoted by $M(\eta, \xi, \phi, g)$. We denote by $\nabla$ the Levi-Civita connection and by $R$ the corresponding Riemann curvature tensor field given by

$$
R(X, Y)=\left[\nabla_{X}, \nabla_{Y}\right]-\nabla_{[X, Y]}
$$

for all vector fields X, Y on $M$. We denote by $S$ the Ricci tensor field of type $(0,2)$, by $Q$ the Ricci operator, that is, the corresponding endomorphism field, and by $r$ the scalar curvature. We define on $M$ the operators $l, h$ and $\tau$ by

$$
l X=R(X, \xi) \xi, \quad h X=\frac{1}{2}\left(\mathscr{L}_{\xi} \phi\right) X, \quad \tau(X, Y)=\left(\mathscr{L}_{\xi} g\right)(X, Y),
$$

where $\mathscr{L}_{\xi}$ is the Lie derivative in the direction of $\xi$. The tensor fields $h, l$ of type $(1,1)$ are self-adjoint and satisfy

$$
h \xi=0, \quad l \xi=0, \quad \operatorname{tr} h=\operatorname{tr} h \phi=0, \quad h \phi=-\phi h .
$$

If $X$ is an eigenvector of $h$ corresponding to the eigenvalue $\lambda$, then $\phi X$ is also an eigenvector of $h$ corresponding to the eigenvalue $-\lambda$, because $h$ anticommutes with $\phi$.

We also have these formulas for a contact metric manifold:

$$
\begin{aligned}
\nabla_{X} \xi & \left.=-\phi X-\phi h X \quad \text { (hence } \nabla_{\xi} \xi=0\right), \\
\nabla_{\xi} \phi & =0 \\
\operatorname{tr} l & =g(Q \xi, \xi)=2 n-\operatorname{tr} h^{2}, \\
\tau & =2 g(\phi \cdot, h \cdot), \\
\nabla_{\xi} \tau & =2 g\left(\phi \cdot, \nabla_{\xi} h \cdot\right), \\
\|\tau\|^{2} & =4 \operatorname{tr} h^{2} .
\end{aligned}
$$

Formulas (2-3)-(2-5) occur in [Blair 1976; 2002], while (2-6) and (2-7) are simple consequences of (2-2) and (2-4). Equation (2-8) arises easily from (2-7); see also [Perrone 1990]. 
A contact metric manifold for which $\xi$ is a Killing vector field is called a $K$ contact manifold. It is well known that a contact metric manifold is $K$-contact if and only if $h=0$.

A contact structure on $M$ gives rise to an almost complex structure on the product $M \times \mathbb{R}$. If this structure is integrable, then the contact metric manifold is said to be Sasakian. Equivalently, a contact metric manifold is Sasakian if and only if

$$
R(X, Y) \xi=\eta(Y) X-\eta(X) Y \text { for all } X, Y \in \mathscr{X}(M) .
$$

On a Sasakian manifold,

$$
\left(\nabla_{X} \phi\right) Y=g(X, Y) \xi-\eta(Y) X \quad \text { for all } X, Y \in \mathscr{X}(M) .
$$

Every Sasakian manifold is $K$-contact, but the converse is true only in the three dimensional case.

The $(\kappa, \mu)$-nullity distribution of a contact metric manifold $M(\eta, \xi, \phi, g)$ for the pair $(\kappa, \mu) \in \mathbb{R}^{2}$ is the distribution

$$
\begin{aligned}
& N(\kappa, \mu): p \rightarrow N_{p}(\kappa, \mu)= \\
& \left\{Z \in T_{p} M \mid R(X, Y) Z=\kappa(g(Y, Z) X-g(X, Z) Y)+\mu(g(Y, Z) h X-g(X, Z) h Y)\right\} .
\end{aligned}
$$

for all $X, Y \in T_{p} M$. So, if the characteristic vector field $\xi$ belongs to the $(\kappa, \mu)$ nullity distribution, then

$$
R(X, Y) \xi=\kappa(\eta(Y) X-\eta(X) Y)+\mu(\eta(Y) h X-\eta(X) h Y)
$$

and the manifold $M$ is called a $(\kappa, \mu)$-contact metric manifold [Blair et al. 1995]. If $\kappa, \mu$ are nonconstant smooth functions on $M$, the manifold $M$ is called generalized $(\kappa, \mu)$-contact metric manifold. It is shown in [Koufogiorgos and Tsichlias 2000] that if $\operatorname{dim} M>3$, then $\kappa$ and $\mu$ are necessarily constants, whereas if $\operatorname{dim} M=3$, such generalized manifolds exist.

Definition 2.1. A $(\kappa, \mu, v)$-contact metric manifold is a $(2 n+1)$-dimensional contact metric manifold $M(\eta, \xi, \phi, g)$ whose curvature tensor satisfies

$$
\begin{aligned}
R(X, Y) \xi=\kappa(\eta(Y) X-\eta(X) Y)+\mu(\eta(Y) h X & -\eta(X) h Y) \\
& +v(\eta(Y) \phi h X-\eta(X) \phi h Y)
\end{aligned}
$$

for all $X, Y \in \mathscr{L}(M)$, where $\kappa, \mu, v$ are smooth functions on $M$.

2.2. Harmonic vector fields. For an $m$-dimensional Riemannian manifold $(M, g)$, we consider the unit tangent sphere bundle $\left(T_{1} M, g_{S}\right)$, where $g_{S}$ is the Sasaki metric. It is known that a unit vector field $V$ can be regarded as an immersion of $M$ into its unit tangent sphere bundle $\left(T_{1} M, g_{S}\right)$. Hence, the induced metric $V^{*} g_{S}$ is given by

$$
\left(V^{*} g_{S}\right)(X, Y)=g(X, Y)+g\left(\nabla_{X} V, \nabla_{Y} V\right) .
$$


We define two tensor fields $A_{V}$ and $L_{V}$ of type $(1,1)$ by

$$
A_{V}=-\nabla V, \quad L_{V}=I+A_{V}^{t} A_{V} .
$$

For a compact oriented manifold $M$, the energy $E(V)$ of $V$ is the energy of the map $V:(M, g) \mapsto\left(T_{1} M, g_{S}\right)$ and is given by

$$
E(V)=\frac{1}{2} \int_{M} \operatorname{tr} L_{V} d v=\frac{m}{2} \operatorname{vol}(M, g)+\frac{1}{2} \int_{M}\|\nabla V\|^{2} d v .
$$

The energy $E(V)$ is equal (up to a constant) to $B(V)=\int_{M}\|\nabla V\|^{2} d v$, which is known as the total bending of $V$ [Wiegmink 1995]. $V$ is called a harmonic vector field if it is a critical point for the energy functional $E$ defined on the set $\mathscr{X}^{1}(M)$. $V$ is a harmonic vector field if and only if the 1 -form $v_{V}$ defined by

$$
v_{V}(X)=\operatorname{tr}\left(Z \mapsto\left(\nabla_{Z} A_{V}^{t}\right) X\right),
$$

vanishes on $V^{\perp}$, that is, on the distribution determined by tangent vectors orthogonal to $V$; see [Wiegmink 1995; Wood 1997; Gil-Medrano 2001]. The map $V:(M, g) \rightarrow\left(T_{1} M, g_{S}\right)$ defines a harmonic map [Han and Yim 1998] if and only if it is a harmonic vector field and if additionally

$$
\left.\operatorname{tr}\left(Z \mapsto R\left(A_{V} Z, V\right) X\right)\right)=0,
$$

for all vector fields $X$ on $M$. Gil-Medrano [2001] introduced similar notions for $M$ being noncompact or nonorientable.

We recall the following:

Definition 2.2 [Perrone 2004]. A contact metric manifold whose characteristic vector field $\xi$ is a harmonic vector field is called an $H$-contact manifold.

\section{Three dimensional $\boldsymbol{H}$-contact metric manifolds}

Here we prove Theorem 1.1, which gives a geometric characterization of the three dimensional $H$-contact metric manifolds in terms of the Riemannian curvature tensor.

Proof. Let us assume that the condition (2-10) is satisfied on $M$. Using (2-1), we have

$$
\begin{aligned}
g(R(\xi, X) Y, Z)= & g(R(Y, Z) \xi, X) \\
= & \kappa g(\eta(Z) Y-\eta(Y) Z, X)+\mu g(\eta(Z) h Y-\eta(Y) h Z, X) \\
& +v g(\eta(Z) \phi h Y-\eta(Y) \phi h Z, X) \\
= & g(\kappa(g(X, Y) \xi-\eta(Y) X)+\mu(g(h X, Y) \xi-\eta(Y) h X) \\
& +v(g(\phi h Y, X) \xi-\eta(Y) \phi h X), Z)
\end{aligned}
$$


for every vector field $Z$ on $M$. So,

$$
\begin{array}{r}
R(\xi, X) Y=\kappa(g(X, Y) \xi-\eta(Y) X)+\mu(g(h X, Y) \xi-\eta(Y) h X) \\
+v(g(\phi h Y, X) \xi-\eta(Y) \phi h X) .
\end{array}
$$

Next, for any $p \in M$, we consider a local orthonormal basis $\{e, \phi e, \xi\}$. The definition of the Ricci operator $Q$ and the relations (2-1), (2-2), and (3-1) imply

$$
\begin{aligned}
Q \xi= & R(\xi, e) e+R(\xi, \phi e) \phi e \\
= & \kappa \xi+\mu g(h e, e) \xi+v g(\phi h e, e) \xi \\
& +\kappa \xi+\mu g(h \phi e, \phi e) \xi+v g(\phi h \phi e, \phi e) \xi=2 \kappa \xi .
\end{aligned}
$$

It is known, see [Perrone 2004, Theorem 3.1] and [González-Dávila and Vanhecke 2001], that a contact metric manifold is an $H$-contact metric manifold if and only if the characteristic vector field $\xi$ is an eigenvector of the Ricci operator $Q$. So, by (3-2) we deduce that $M$ is an $H$-contact metric manifold.

Conversely, we assume that the characteristic vector field $\xi$ is an eigenvector of the Ricci operator $Q$. Let $U$ be the open subset of $M$ where $h \neq 0$, and let $V$ be the open subset of points $m \in M$ such that $h=0$ in a neighborhood of $m$. Then $U \cup V$ is an open and dense subset of $M$. For every $p \in U$ there exists an open neighborhood of $p$ such that $h e=\lambda e$ and $h \phi e=-\lambda \phi e$, where $\lambda$ is a nonvanishing smooth function. So, for every point $m \in U \cup V$ there exists a local orthonormal basis $\{\xi, e, \phi e\}$ of smooth eigenvectors of $h$ in a neighborhood of $m$. We need this lemma:

Lemma 3.1 [Calvaruso et al. 1999]. On $U$ the following relations are true:

$$
\begin{array}{rlrl}
\nabla_{\xi} e & =-\alpha \phi e, & \nabla_{\xi} \phi e & =\alpha e, \\
\nabla_{e} \xi & =-(\lambda+1) \phi e, & \nabla_{\phi e} \xi=-(\lambda-1) e, \\
\nabla_{e} e & =\frac{1}{2 \lambda}((\phi e)(\lambda)+A) \phi e, & \nabla_{\phi e} \phi e=\frac{1}{2 \lambda}(e(\lambda)+B) e, \\
\nabla_{e} \phi e & =-\frac{1}{2 \lambda}((\phi e)(\lambda)+A) e+(\lambda+1) \xi, & & \\
\nabla_{\phi e} e & =-\frac{1}{2 \lambda}(e(\lambda)+B) \phi e+(\lambda-1) \xi, & &
\end{array}
$$

and

$$
\nabla_{\xi} h=2 \alpha h \phi+\xi(\lambda) s,
$$

where $\alpha$ is a smooth function, $A=S(\xi, e), B=S(\xi, \phi e)$, and $s$ is the tensor field of type $(1,1)$ defined by $s \xi=0$, se $=e$, and $s \phi e=-\phi e$.

If the open set $V$ is nonempty, then it inherits the contact structure of $M$. In particular, the structure is Sasakian and the theorem is trivial on $V$. Next, let $U$ be a nonempty set and let $\{\xi, e, \phi e\}$ be the local $\phi$-basis described above. Then 
it is proved in [Perrone 1996], that the Ricci operator on a 3-dimensional contact metric manifold is given by

$$
Q=a I+\beta \eta \otimes \xi+\phi \nabla_{\xi} h-\sigma\left(\phi^{2}\right) \otimes \xi+\sigma(e) \eta \otimes e+\sigma(\phi e) \eta \otimes \phi e,
$$

where $\sigma=\left.S(\xi, \cdot)\right|_{\operatorname{ker} \eta}, a=r / 2-1+\lambda^{2}$, and $\beta=-r / 2+3-3 \lambda^{2}$. Using (3-3), we get

$$
Q=a I+\beta \eta \otimes \xi+2 \alpha h+\xi(\lambda) \phi s-\sigma\left(\phi^{2}\right) \otimes \xi+\sigma(e) \eta \otimes e+\sigma(\phi e) \eta \otimes \phi e .
$$

By [Perrone 2004] we deduce easily that the characteristic vector field $\xi$ is a harmonic vector field if and only if $\sigma=0$. Using now the relations $s=(1 / \lambda) h, \sigma=0$, and (3-4), we deduce that

$$
Q=a I+\beta \eta \otimes \xi+2 \alpha h+\frac{\xi(\lambda)}{\lambda} \phi h .
$$

On the other hand, (2-5) implies that $\operatorname{tr} l=2\left(1-\lambda^{2}\right)$. Moreover, in Riemannian 3-manifolds the Ricci operator determines completely the curvature tensor by the formula [O’Neill 1983]

$$
\begin{aligned}
& R(X, Y) Z=g(Y, Z) Q X-g(X, Z) Q Y+g(Q Y, Z) X \\
& \quad-g(Q X, Z) Y-\frac{r}{2}(g(Y, Z) X-g(X, Z) Y) .
\end{aligned}
$$

Setting $Z=\xi$ and using the fact that $\xi$ is an eigenvector of $Q$, we obtain that

$$
R(X, Y) \xi=\eta(Y) Q X-\eta(X) Q Y+(\operatorname{tr} l-r / 2)(\eta(Y) X-\eta(X) Y) .
$$

Substituting (3-5) in the last equation, we derive

$$
\begin{aligned}
R(X, Y) \xi=\left(1-\lambda^{2}\right)(\eta(Y) X-\eta(X) Y)+2 \alpha(\eta(Y) h X-\eta(X) h Y) & \\
& +\frac{\xi(\lambda)}{\lambda}(\eta(Y) \phi h X-\eta(X) \phi h Y),
\end{aligned}
$$

which is the relation (2-10) with $\kappa=1-\lambda^{2}=(\operatorname{tr} l) / 2, \mu=2 \alpha$, and $v=\xi(\lambda) / \lambda$ on $U$. The proof is completed.

Remark 3.1. Theorem 1.1 generalizes [Perrone 2003, Theorem 1.1], which gives a geometric interpretation of generalized $(\kappa, \mu)$-contact metric manifolds in terms of harmonic maps.

Following similar steps as in the proof of the Theorem 1.1 and using that on a Sasakian manifold the Ricci tensor $S$ is given by

$$
S=(r / 2-1) g+(-r / 2+3) \eta \otimes \eta,
$$

as in [Perrone 1990], one could easily prove this proposition: 
Proposition 3.1. Let $M(\eta, \xi, \phi, g)$ be a contact metric 3-manifold. Then these conditions are equivalent on $U \cup V$ :

(1) $\left.S(\xi, \cdot)\right|_{\text {ker } \eta}=0$.

(2) $\quad R(X, Y) \xi=\kappa(\eta(Y) X-\eta(X) Y)+\mu(\eta(Y) h X-\eta(X) h Y)$

$$
+v(\eta(Y) \phi h X-\eta(X) \phi h Y)
$$

where $\kappa, \mu, v$ are differentiable functions on $U \cup V$.

(3) $Q \phi-\phi Q=2 v \phi h-2 \mu h$.

(4) $Q=(r / 2-\kappa) I+(-r / 2+3 \kappa) \eta \otimes \xi+\mu h+v \phi h$.

Proposition 3.1 could be considered as a generalization of the main theorem of [Koufogiorgos 1995] in that the constants that appear there are substituted by differentiable functions. The formula for $Q$ has additional terms and extends the notion of $\eta$ - Einstein contact metric manifolds [Blair et al. 1990, Proposition 3.2].

Let $M(\eta, \xi, \phi, g)$ be a contact metric 3-manifold. A D-homothetic transformation [Tanno 1968] is the transformation

$$
\bar{\eta}=\alpha \eta, \quad \bar{\xi}=\frac{1}{\alpha} \xi, \quad \bar{\phi}=\phi, \quad \bar{g}=\alpha g+\alpha(\alpha-1) \eta \otimes \eta
$$

at the structure tensors, where $\alpha$ is a positive constant. It is well known [Tanno $1968]$ that $M(\bar{\eta}, \bar{\xi}, \bar{\phi}, \bar{g})$ is also a contact metric manifold. When two contact structures $(\eta, \xi, \phi, g)$ and $(\bar{\eta}, \bar{\xi}, \bar{\phi}, \bar{g})$ are related by (3-7), we will say that they are $D$-homothetic.

Proposition 3.2. If $M(\eta, \xi, \phi, g)$ is a 3-dimensional ( $\kappa, \mu, v)$-contact metric manifold, then $M(\bar{\eta}, \bar{\xi}, \bar{\phi}, \bar{g})$ is also a $(\kappa, \mu, v)$-contact metric manifold.

Proof. By direct computations we easily see that the tensor $h$ and the curvature tensor transform as $\bar{h}=(1 / \alpha) h$, and

$$
\begin{aligned}
\alpha \bar{R}(X, Y) \bar{\xi}= & R(X, Y) \xi+(\alpha-1)^{2}(\eta(Y) X-\eta(X) Y) \\
& \left.-(\alpha-1)\left(\left(\nabla_{X} \phi\right)\right) Y-\left(\nabla_{Y} \phi\right) X+\eta(X)(Y+h Y)-\eta(Y)(X+h X)\right) .
\end{aligned}
$$

It is well known, for example, [Blair 2002, page 74] or [Tanno 1989], that every 3dimensional contact metric manifold is a contact strongly pseudoconvex integrable CR manifold or, equivalently, satisfies the integrability condition

$$
\left(\nabla_{X} \phi\right) Y=g(X+h X, Y) \xi-\eta(Y)(X+h X) .
$$

Using the relations above we finally obtain that

$$
\begin{array}{r}
\bar{R}(X, Y) \bar{\xi}=\frac{\kappa+\alpha^{2}-1}{\alpha^{2}}(\bar{\eta}(Y) X-\bar{\eta}(X) Y)+\frac{\mu+2(\alpha-1)}{\alpha}(\bar{\eta}(Y) \bar{h} X-\bar{\eta}(X) \bar{h} Y) \\
+\frac{v}{\alpha}(\bar{\eta}(Y) \bar{\phi} \bar{h} X-\bar{\eta}(X) \bar{\phi} \bar{h} Y)
\end{array}
$$


for all vector fields $X$ and $Y$ on $M$. Thus $M(\bar{\eta}, \bar{\xi}, \bar{\phi}, \bar{g})$ is a $(\bar{\kappa}, \bar{\mu}, \bar{v})$-contact metric manifold with

$$
\bar{\kappa}=\frac{\kappa+\alpha^{2}-1}{\alpha^{2}}, \quad \bar{\mu}=\frac{\mu+2 \alpha-2}{\alpha}, \quad \bar{v}=\frac{v}{\alpha} .
$$

A natural question is, Do there exist contact metric manifolds that satisfy (2-10) when $\kappa, \mu, v$ are nonconstant smooth functions and the vector fields $X, Y$ are arbitrary?

\section{4. $(\kappa, \mu, v)$-contact metric manifolds}

We construct some examples of 3-dimensional $(\kappa, \mu, v)$-contact metric manifolds.

Example 4.1. Consider the 3-dimensional manifold

$$
M=\left\{(x, y, z) \in \mathbb{R}^{3} \mid x>0, y>0, z>0\right\},
$$

where $(x, y, z)$ are the cartesian coordinates in $\mathbb{R}^{3}$. We define three vector fields on $M$ as

$$
e_{1}=\frac{\partial}{\partial x}, \quad e_{2}=\frac{\partial}{\partial y}, \quad e_{3}=-\frac{4}{z} e^{G} G_{y} \frac{\partial}{\partial x}+\beta \frac{\partial}{\partial y}+e^{G / 2} \frac{\partial}{\partial z},
$$

where $G=G(y, z)<0$ for all $(y, z)$ is a solution of the partial differential equation

$$
2 G_{y y}+G_{y}^{2}=-z e^{-G},
$$

and the function $\beta=\beta(x, y, z)$ solves the system of partial differential equations

$$
\begin{aligned}
& \beta_{x}=\frac{4}{z x^{2}} e^{G}, \\
& \beta_{y}=\frac{1}{2 z} e^{G / 2}-\frac{G_{z} e^{G / 2}}{2}-\frac{4 e^{G} G_{y}}{x z} .
\end{aligned}
$$

The vector fields $e_{1}, e_{2}, e_{3}$ are linearly independent at each point of $M$. We define a Riemannian metric $g$ on $M$ such that $g\left(e_{i}, e_{j}\right)=\delta_{i j}$ for $i, j=1,2,3$. We easily get that

(4-3) $\quad\left[e_{1}, e_{2}\right]=0$,

(4-4) $\left[e_{2}, e_{3}\right]=2 e_{1}+\left(\frac{1}{2 z} e^{G / 2}-\frac{G_{z}}{2} e^{G / 2}-\frac{4}{x z} e^{G} G_{y}-\frac{\beta G_{y}}{2}\right) e_{2}+\frac{1}{2} G_{y} e_{3}$,

(4-5) $\quad\left[e_{1}, e_{3}\right]=\frac{4 e^{G}}{z x^{2}} e_{2}$.

Let $\eta$ be the 1 -form defined by $\eta(W)=g\left(W, e_{1}\right)$ for all $W \in \mathscr{X}(M)$. Then $\eta$ is a contact form since $\eta \wedge d \eta \neq 0$ everywhere on $M$. Let $\phi$ be the tensor field of type $(1,1)$ defined by $\phi e_{1}=0, \phi e_{2}=e_{3}$, and $\phi e_{3}=-e_{2}$. Using the linearity 
of $\phi, d \eta$, and $g$, we easily obtain that $\eta\left(e_{1}\right)=1, d \eta(Z, W)=g(\phi Z, W)$, and $g(\phi Z, \phi W)=g(Z, W)-\eta(Z) \eta(W)$ for all vector fields $Z, W$ on $M$. Hence $M\left(\eta, e_{1}, \phi, g\right)$ is a contact metric manifold. Let $\nabla$ be the Levi-Civita connection corresponding to $g$, and let $R$ be the Riemannian curvature tensor of $g$.

Setting $\xi=e_{1}, X=e_{2}$, and $\phi X=e_{3}$ and using Koszul's formula

$$
\begin{aligned}
2 g\left(\nabla_{Y} Z, W\right)=Y g(Z, W)+Z g(W, Y)-W g(Y, Z) & \\
& -g(Y,[Z, W])-g(Z,[Y, W])+g(W,[Y, Z])
\end{aligned}
$$

and also (4-1) and (4-2), we calculate

$$
\begin{array}{ccc}
\nabla_{X} \xi=\left(-\frac{2 e^{G}}{z x^{2}}-1\right) \phi X, & \nabla_{\phi X} \xi=\left(1-\frac{2 e^{G}}{z x^{2}}\right) X & \nabla_{\phi X} \phi X=\frac{1}{2} G_{y} X, \\
\nabla_{\xi} X=-\left(1+\frac{2 e^{G}}{z x^{2}}\right) \phi X, & \nabla_{\xi} \phi X=\left(1+\frac{2 e^{G}}{z x^{2}}\right) X, & \nabla_{\xi} \xi=0, \\
\nabla_{\phi X} X=-\frac{1}{2} G_{y} \phi X+\left(\frac{2 e^{G}}{z x^{2}}-1\right) \xi \\
\nabla_{X} X=\left(-\frac{1}{2 z} e^{G / 2}+\frac{G_{z}}{2} e^{G / 2}+\frac{4}{x z} e^{G} G_{y}+\frac{\beta G_{y}}{2}\right) \phi X, \\
\nabla_{X} \phi X=\left(\frac{1}{2 z} e^{G / 2}-\frac{G_{z}}{2} e^{G / 2}-\frac{4}{x z} e^{G} G_{y}-\beta \frac{G_{y}}{2}\right) X+\left(\frac{2 e^{G}}{z x^{2}}+1\right) \xi .
\end{array}
$$

From the definition of the tensor field $h$ and relations (4-3) and (4-5) we get that $h \xi=0$ and

$$
h X=\frac{1}{2}\left(\mathscr{L}_{\xi} \phi\right) X=\frac{1}{2}([\xi, \phi X]-\phi[\xi, X])=\frac{2 e^{G}}{z x^{2}} X .
$$

Similarly, we obtain that

$$
h \phi X=-\frac{2 e^{G}}{z x^{2}} \phi X .
$$

Setting now $\kappa=1-\left(4 e^{2 G}\right) /\left(z^{2} x^{4}\right), \mu=2\left(1+\left(2 e^{G}\right) /\left(z x^{2}\right)\right)$, and $v=-2 / x$ and using the last two relations, we easily deduce that

$$
\begin{aligned}
R(X, \xi) \xi & =\frac{-4 e^{G}}{z x^{3}} \phi X+\left(1+\frac{2 e^{G}}{z x^{2}}\right)^{2} X \\
& =\kappa(\eta(\xi) X-\eta(X) \xi)+\mu(\eta(\xi) h X-\eta(X) h \xi)
\end{aligned}
$$

$+v(\eta(\xi) \phi h X-\eta(X) \phi h \xi)$,

$$
\begin{aligned}
R(\phi X, \xi) \xi & =\frac{-4 e^{G}}{z x^{3}} X-\left(1+\frac{2 e^{G}}{z x^{2}}\right)\left(\frac{6 e^{G}}{z x^{2}}-1\right) \\
& =\kappa(\eta(\xi) \phi X-\eta(\phi X) \xi)+\mu(\eta(\xi) h \phi X-\eta(\phi X) h \xi)
\end{aligned}
$$




$$
\begin{aligned}
R(X, \phi X) \xi=0=\kappa(\eta(\phi X) X-\eta(X) \phi X)+\mu( & \eta(\phi X) h X-\eta(X) h \phi X) \\
& +v(\eta(\phi X) \phi h X-\eta(X) \phi h \phi X) .
\end{aligned}
$$

By direct calculation, these relations yield

$$
\begin{aligned}
R(Z, W) \xi=\kappa[\eta(W) Z-\eta(Z) W]+\mu[\eta(W) h Z-\eta(Z) h W] & \\
& +v[\eta(W) \phi h Z-\eta(Z) \phi h W]
\end{aligned}
$$

for all vector fields $Z, W$, on $M$, where $\kappa, \mu, v$ are nonconstant smooth functions. Hence, it has been shown that $M$ is a $(\kappa, \mu, v)$-contact metric manifold.

Remark 4.1. In particular, a solution of (4-1) is given by

$$
y=4 \sqrt{\frac{\pi}{z}} \operatorname{erf}\left(\sqrt{-\frac{G}{2}}\right)
$$

where $\operatorname{erf}(x)=\frac{1}{\sqrt{2 \pi}} \int_{0}^{x} e^{-t^{2}} d t$. This solution justifies restricting $y$ and $z$ to be positive.

Example 4.2. We consider the 3-dimensional manifold $M=\left\{(x, y, z) \in \mathbb{R}^{3} \mid z>0\right\}$ and the vector fields

$e_{1}=\frac{\partial}{\partial x}, \quad e_{2}=\frac{\partial}{\partial y}, \quad e_{3}=(2 y+2 z) \frac{\partial}{\partial x}+\left(-\left(\frac{y}{2} c+z c+\frac{1}{2 z}\right) y+\frac{1}{c} e^{c x} z\right) \frac{\partial}{\partial y}+\frac{\partial}{\partial z}$,

where $c$ is a nonzero real constant. Let $\eta$ be the 1 -form dual to $e_{1}$. We define the structure tensors $\xi$ and $\phi$ by $\xi=e_{1}$ and $\phi e_{1}=0, \phi e_{2}=e_{3}$, and $\phi e_{3}=-e_{2}$. Let $g$ be the Riemannian metric defined by $g\left(e_{i}, e_{j}\right)=\delta_{i j}$ for $i, j=1,2,3$. Following the same procedure as in the previous example, we finally deduce that $M(\eta, \xi, \phi, g)$ is also a $(\kappa, \mu, v)$-contact metric manifold with $\kappa=1-\left(e^{2 c x} z^{2}\right) /(4), \mu=2+e^{c x} z$, and $v=c$.

Example 4.3. We consider the 3-dimensional manifold

$$
M=\left\{(x, y, z) \in \mathbb{R}^{3} \mid 2 x+e^{y+z}>0, y \neq z\right\}
$$

and the vector fields $e_{1}=\partial / \partial x$,

$$
\begin{aligned}
e_{2}=\left(-\left(\frac{y^{2}+z^{2}}{2}\right)\left(2 x+e^{y+z}\right)^{1 / 2}\right) \frac{\partial}{\partial x} & +\left(\frac{z\left(2 x+e^{y+z}\right)^{1 / 2}}{y-z}+\frac{\left(2 x+e^{y+z}\right)^{-1 / 2}}{y-z}\right) \frac{\partial}{\partial y} \\
& +\left(\frac{y\left(2 x+e^{y+z}\right)^{1 / 2}}{z-y}+\frac{\left(2 x+e^{y+z}\right)^{-1 / 2}}{z-y}\right) \frac{\partial}{\partial z}, \\
e_{3}=\left(\left(\frac{y^{2}+z^{2}}{2}\right)\left(2 x+e^{y+z}\right)^{1 / 2}\right) \frac{\partial}{\partial x}+ & \left(\frac{z\left(2 x+e^{y+z}\right)^{1 / 2}}{z-y}+\frac{\left(2 x+e^{y+z}\right)^{-1 / 2}}{y-z}\right) \frac{\partial}{\partial y} \\
& +\left(\frac{y\left(2 x+e^{y+z}\right)^{1 / 2}}{y-z}+\frac{\left(2 x+e^{y+z}\right)^{-1 / 2}}{z-y}\right) \frac{\partial}{\partial z} .
\end{aligned}
$$


Let $\eta$ be the 1-form dual to $e_{1}$. We define the structure tensors $\xi$ and $\phi$ by $\xi=e_{1}$ and $\phi e_{1}=0, \phi e_{2}=e_{3}$, and $\phi e_{3}=-e_{2}$. Let $g$ be the Riemannian metric defined by $g\left(e_{i}, e_{j}\right)=\delta_{i j}$ for $i, j=1,2,3$. Following the same procedure as in the previous examples, we finally deduce that $M(\eta, \xi, \phi, g)$ is also a $(\kappa, \mu, v)$-contact metric manifold with $\kappa=1-1 /\left(2 x+e^{y+z}\right)^{2}, \mu=2$, and $\nu=-2 /\left(2 x+e^{y+z}\right)$.

Remark 4.2. By choosing any positive number $\alpha$, relations (3-8) imply that we can construct a family of $(\kappa, \mu, v)$-contact metric manifolds depending on the parameter $\alpha$.

Remark 4.3. To our knowledge, the examples above are the first of contact metric manifolds in which the Reeb vector fields are harmonic vector fields but don't define harmonic maps.

Let $M(\eta, \xi, \phi, g)$ be a $(2 n+1)$-dimensional $(\kappa, \mu, v)$-contact metric manifold and $B=\{p \in M \mid \kappa(p)=1\}$. Then the set $N=M \backslash B$ is an open subset of $M$ and hence inherits the contact structure of $M$, that is, $N(\eta, \xi, \phi, g)$ is a contact metric manifold that satisfies (2-10) with $\kappa<1$ everywhere.

Now, it is remarkable that the following theorem is valid.

Theorem 4.1. Every $(\kappa, \mu, v)$-contact metric manifold $M(\eta, \xi, \phi, g)$ of dimension greater than 3 is either a Sasakian manifold or a $(\kappa, \mu)$-contact metric manifold, that is, the functions $\kappa$ and $\mu$ are constants and $v$ is the zero function.

Here and in what follows we denote the $(\kappa, \mu, 0)$-contact metric manifolds by $(\kappa, \mu)$-contact metric manifolds.

The proof of this theorem depends largely on the next three lemmas.

Lemma 4.2. The following relations are satisfied on every $(2 n+1)$-dimensional $(\kappa, \mu, \nu)$-contact metric manifold $M(\eta, \xi, \phi, g)$.

$$
\begin{aligned}
l & =-\kappa \phi^{2}+\mu h+v \phi h, \\
l \phi-\phi l= & 2 \mu h \phi+2 v h, \\
h^{2}= & (\kappa-1) \phi^{2} \quad \text { for } \kappa \leq 1, \\
\nabla_{\xi} h= & \mu h \phi+v h, \\
R(\xi, X) Y= & \kappa(g(X, Y) \xi-\eta(Y) X)+\mu(g(h X, Y) \xi-\eta(Y) h X) \\
& +v(g(\phi h Y, X) \xi-\eta(Y) \phi h X), \\
Q \xi= & (2 n \kappa) \xi, \\
\left(\nabla_{X} \phi\right) Y= & g(X+h X, Y) \xi-\eta(Y)(X+h X),
\end{aligned}
$$




$$
\begin{aligned}
(4-13) \quad\left(\nabla_{X} h\right) Y-\left(\nabla_{Y} h\right) X= & (1-\kappa)(\eta(X) \phi Y-\eta(Y) \phi X+2 g(X, \phi Y) \xi) \\
& +(1-\mu)(\eta(X) \phi h Y-\eta(Y) \phi h X) \\
& +v(\eta(X) h Y-\eta(Y) h X), \\
\xi(\kappa)= & 2 v(\kappa-1), \\
(4-14) \quad\left(\nabla_{X} \phi h\right) Y-\left(\nabla_{Y} \phi h\right) X= & (1-\kappa)(\eta(Y) X-\eta(X) Y) \\
& +(1-\mu)(\eta(Y) h X-\eta(X) h Y) \\
& +v(\eta(X) \phi h Y-\eta(Y) \phi h X)
\end{aligned}
$$

for all vector fields $X$ and $Y$ on $M$.

Proof. The proof of (4-6)-(4-11) and (4-13) is similar to that of [Blair et al. 1995, Lemma 3.1]. Following the proof of [Equation (3.9)(v)] of that lemma, we obtain

$$
(\kappa-1)\left(\left(\nabla_{X} \phi\right) Y-g(X+h X, Y) \xi+\eta(Y)(X+h X)\right)=0 .
$$

The last equation shows that Equation (4-12) is valid on $N$. The set $B$ is a closed subset of $M$. Since the interior $B^{\circ}$ of $B$ is a Sasakian manifold and using (2-9), we deduce that (4-12) is valid on $B^{\circ}$. Next, for all $X, Y \in \mathscr{L}(M)$ we consider the function $F(X, Y): M \mapsto \mathbb{R}$ given by

$$
F(X, Y)(p)=\left(\left(\nabla_{X} \phi\right) Y-g(X+h X, Y) \xi+\eta(Y)(X+h X)\right)(p)
$$

for all $p \in M$. Since the continuous function $F(X, Y)$ vanishes on $N$ and $B^{\circ}$, we get that this function vanishes on every point of $M$. Hence, the proof of (4-12) has been completed. Using (2-2), (4-8), and (4-9), we obtain

$$
\nabla_{\xi} h^{2}=\left(\nabla_{\xi} h\right) h+h\left(\nabla_{\xi} h\right)=(\mu h \phi+v h) h+h(\mu h \phi+v h)=2 v(\kappa-1) \phi^{2} .
$$

Alternately, differentiating (4-8) along $\xi$ and using (2-4), we get $\nabla_{\xi} h^{2}=\xi(\kappa) \phi^{2}$. This equation with the one before completes the proof of (4-14). The relation (4-15) is an immediate consequence of (4-12), (4-13), and

$$
\left(\nabla_{X} \phi h\right) Y-\left(\nabla_{Y} \phi h\right) X=\left(\nabla_{X} \phi\right) h Y-\left(\nabla_{Y} \phi\right) h X+\phi\left(\left(\nabla_{X} h\right) Y-\left(\nabla_{Y} h\right) X\right) .
$$

Lemma 4.3. The following differential equation is satisfied on every $(\kappa, \mu, v)$ contact metric manifold $M(\eta, \xi, \phi, g)$ of dimension $(2 n+1)$ :

$$
\begin{aligned}
& 0=\xi(\kappa)[\eta(Y) X-\eta(X) Y]+\xi(\mu)[\eta(Y) h X-\eta(X) h Y] \\
& \quad+\xi(v)[\eta(Y) \phi h X-\eta(X) \phi h Y]-X(\kappa) \phi^{2} Y+Y(\kappa) \phi^{2} X \\
& \quad+X(\mu) h Y-Y(\mu) h X+X(v) \phi h Y-Y(v) \phi h X
\end{aligned}
$$


Proof. Differentiating (2-10) along an arbitrary vector field $Z$ and using (2-3) we get

$\nabla_{Z} R(X, Y) \xi=$

$Z(\kappa)(\eta(Y) X-\eta(X) Y)+Z(\mu)(\eta(Y) h X-\eta(X) h Y)+Z(v)(\eta(Y) \phi h X-\eta(X) \phi h Y)$ $+\kappa\left(\left(\eta\left(\nabla_{Z} Y\right)-g(Y, \phi Z)-g(Y, \phi h Z)\right) X+\eta(Y) \nabla_{Z} X\right.$

$$
\left.-\left(\left(\eta\left(\nabla_{Z} X\right)-g(X, \phi Z)-g(X, \phi h Z)\right) Y+\eta(X) \nabla_{Z} Y\right)\right)
$$

$+\mu\left(\left(\eta\left(\nabla_{Z} Y\right)-g(Y, \phi Z)-g(Y, \phi h Z)\right) h X+\eta(Y) \nabla_{Z} h X\right.$

$$
\left.-\left(\left(\eta\left(\nabla_{Z} X\right)-g(X, \phi Z)-g(X, \phi h Z)\right) h Y+\eta(X) \nabla_{Z} h Y\right)\right)
$$

$+v\left(\left(\eta\left(\nabla_{Z} Y\right)-g(Y, \phi Z)-g(Y, \phi h Z)\right) \phi h X+\eta(Y) \nabla_{Z} \phi h X\right.$

$$
\left.-\left(\left(\eta\left(\nabla_{Z} X\right)-g(X, \phi Z)-g(X, \phi h Z)\right) \phi h Y+\eta(X) \nabla_{Z} \phi h Y\right)\right) \text {. }
$$

Now, using (2-3), (2-10), and the last relation, we deduce

$$
\left(\nabla_{Z} R\right)(X, Y, \xi)=\nabla_{Z} R(X, Y) \xi-R\left(\nabla_{Z} X, Y\right) \xi-R\left(X, \nabla_{Z} Y\right) \xi-R(X, Y) \nabla_{Z} \xi
$$

$$
=
$$

$Z(\kappa)(\eta(Y) X-\eta(X) Y)+Z(\mu)(\eta(Y) h X-\eta(X) h Y)+Z(v)(\eta(Y) \phi h X-\eta(X) \phi h Y)$

$+\kappa(-g(Y, \phi Z+\phi h Z) X+g(X, \phi Z+\phi h Z) Y)$

$+\mu(-g(Y, \phi Z+\phi h Z) h X+g(X, \phi Z+\phi h Z) h Y$

$$
\left.+\eta(Y)\left(\nabla_{Z} h\right) X-\eta(X)\left(\nabla_{Z} h\right) Y\right)
$$

$+v(-g(Y, \phi Z+\phi h Z) \phi h X+g(X, \phi Z+\phi h Z) \phi h Y$

$$
\left.+\eta(Y)\left(\nabla_{Z} \phi h\right) X-\eta(X)\left(\nabla_{Z} \phi h\right) Y\right)
$$

$+R(X, Y) \phi Z+R(X, Y) \phi h Z$.

Using the last relation and the second Bianchi identity

$$
\left(\nabla_{Z} R\right)(X, Y, \xi)+\left(\nabla_{X} R\right)(Y, Z, \xi)+\left(\nabla_{Y} R\right)(Z, X, \xi)=0
$$

we get

$Z(\kappa)(\eta(Y) X-\eta(X) Y)+Z(\mu)(\eta(Y) h X-\eta(X) h Y)+Z(v)(\eta(Y) \phi h X-\eta(X) \phi h Y)$ $+$

$X(\kappa)[\eta(Z) Y-\eta(Y) Z]+X(\mu)[\eta(Z) h Y-\eta(Y) h Z]+X(v)[\eta(Z) \phi h Y-\eta(Y) \phi h Z]$ $+$

$Y(\kappa)[\eta(X) Z-\eta(Z) X]+Y(\mu)[\eta(X) h Z-\eta(Z) h X]+Y(\nu)[\eta(X) \phi h Z-\eta(Z) \phi h X]$ $+2 \kappa[g(\phi Y, Z) X+g(\phi Z, X) Y+g(\phi X, Y) Z]$ 


$$
\begin{aligned}
& +\mu\left(2 g(\phi Y, Z) h X+\eta(Z)\left(\left(\nabla_{X} h\right) Y-\left(\nabla_{Y} h\right) X\right)\right. \\
& +2 g(\phi Z, X) h Y+\eta(X)\left(\left(\nabla_{Y} h\right) Z-\left(\nabla_{Z} h\right) Y\right) \\
& \left.+2 g(\phi X, Y) h Z+\eta(Y)\left(\left(\nabla_{Z} h\right) X-\left(\nabla_{X} h\right) Z\right)\right) \\
& +v\left(2 g(\phi Y, Z) \phi h X+\eta(Z)\left(\left(\nabla_{X} \phi h\right) Y-\left(\nabla_{Y} \phi h\right) X\right)\right. \\
& +2 g(\phi Z, X) \phi h Y+\eta(X)\left(\left(\nabla_{Y} \phi h\right) Z-\left(\nabla_{Z} \phi h\right) Y\right) \\
& \left.+2 g(\phi X, Y) \phi h Z+\eta(Y)\left(\left(\nabla_{Z} \phi h\right) X-\left(\nabla_{X} \phi h\right) Z\right)\right) \\
& +R(X, Y) \phi Z+R(Y, Z) \phi X+R(Z, X) \phi Y \\
& +R(X, Y) \phi h Z+R(Y, Z) \phi h X+R(Z, X) \phi h Y=0 .
\end{aligned}
$$

for all $X, Y, Z \in \mathscr{X}(M)$. Setting $\xi$ instead of $Z$ in the last equation, we finally obtain

$$
\begin{gathered}
\xi(\kappa)(\eta(Y) X-\eta(X) Y)+\xi(\mu)(\eta(Y) h X-\eta(X) h Y)+\xi(v)(\eta(Y) \phi h X-\eta(X) \phi h Y) \\
+\mu \eta(Y)\left\{\left(\nabla_{\xi} h\right) X-\left(\nabla_{X} h\right) \xi\right\}+v \eta(Y)\left\{\left(\nabla_{\xi} \phi h\right) X-\left(\nabla_{X} \phi h\right) \xi\right\} \\
+\mu \eta(X)\left\{\left(\nabla_{Y} h\right) \xi-\left(\nabla_{\xi} h\right) Y\right\}+v \eta(X)\left\{\left(\nabla_{Y} \phi h\right) \xi-\left(\nabla_{\xi} \phi h\right) X\right\} \\
+\mu\left\{\left(\nabla_{X} h\right) Y-\left(\nabla_{Y} h\right) X\right\} \quad+v\left\{\left(\nabla_{X} \phi h\right) Y-\left(\nabla_{Y} \phi h\right) X\right\} \\
-X(\kappa) \phi^{2} Y+X(\mu) h Y+X(v) \phi h Y-Y(\mu) h X-Y(v) \phi h X+Y(\kappa) \phi^{2} X \\
+R(Y, \xi) \phi X+R(Y, \xi) \phi h X+R(\xi, X) \phi Y+R(\xi, X) \phi h Y=0 .
\end{gathered}
$$

Substituting (4-10), (4-13), and (4-15) in the last relation we deduce (4-16).

By mimicking the proof of [Koufogiorgos and Tsichlias 2000, Lemma 3.4], we have

Lemma 4.4. For every $p \in N$, there exists an open neighborhood $W$ of $p$ and orthonormal local vector fields $X_{i}, \phi X_{i}$, and $\xi$ for $i=1, \ldots, n$, defined on $W$, such that

$$
h X_{i}=\lambda X_{i}, \quad h \phi X_{i}=-\lambda \phi X_{i}, \quad h \xi=0,
$$

for $i=1, \ldots, n$, where $\lambda=\sqrt{1-\kappa}$.

Proof of Theorem 4.1. Let $p \in N$, and suppose below that the indices $i$ and $j$ assume the range $1, \ldots, n$ unless otherwise restricted. Then Lemma 4.4 implies the existence of a local orthonormal basis $\left\{X_{i}, \phi X_{i}, \xi\right\}$ on $W$ that satisfies the relations (4-17). Substituting $X=X_{i}$ and $Y=\phi X_{i}$ in (4-16), we get that

$$
\begin{aligned}
X_{i}(\kappa)-\lambda X_{i} \mu-\lambda \phi X_{i} v & =0, \\
-\phi X_{i}(\kappa)-\lambda \phi X_{i}(\mu)+\lambda X_{i}(v) & =0 .
\end{aligned}
$$

Replacing $X$ and $Y$ by $X_{i}$ and $X_{j}$, respectively, for $i \neq j$, Equation (4-16) gives

$$
X_{i}(\kappa)+\lambda X_{i}(\mu)=0 \quad \text { and } \quad X_{i}(\nu)=0 .
$$


Finally, substituting $X=\phi X_{i}$ and $Y=\phi X_{j}$ for $i \neq j$ in (4-16), we obtain

$$
\phi X_{i}(\kappa)-\lambda \phi X_{i}(\mu)=0 \text { and } \phi X_{i}(\nu)=0 .
$$

Combining (4-18), (4-19), and (4-20), we deduce that

$$
X_{i}(\kappa)=\phi X_{i}(\kappa)=X_{i}(\mu)=\phi X_{i}(\mu)=X_{i}(\nu)=\phi X_{i}(\nu)=0 .
$$

Using (4-21) we get $d \mu=\xi(\mu) \eta$, and so

$$
0=d(d \mu)=d \xi(\mu) \wedge \eta+\xi(\mu) d \eta .
$$

Acting (4-22) on the pairs $\left(X_{i}, \xi\right)$ and $\left(\phi X_{i}, \xi\right)$, respectively, we get that

$$
d \xi(\mu)=\xi \xi(\mu) \eta
$$

Substituting (4-23) into (4-22), we get $\xi(\mu)=0$, that is, the function $\mu$ is a constant on every connected component of $W$. Following the same procedure, it can be shown that the functions $\kappa, v$ are also constant on every connected component of $W$. If the connected manifold $M$ contains points for which $\kappa=1$, then due to the continuity of the function $\kappa$, we have that $\kappa=1$ everywhere on $M$. So, by (2-10) and (4-8) we deduce that $R(X, Y) \xi=\eta(Y) X-\eta(X) Y$ for all $X, Y \in \mathscr{L}(M)$, that is, $M$ is a Sasakian manifold. If $\kappa<1$ everywhere on $M$, that is, $N=M$, then we easily get that the continuous functions $\kappa, \mu, v$ are constant on $M$. Also, relation (4-14) and the assumption that $\kappa<1$ on $M$ imply that $v=0$ on $M$. Therefore the condition (2-10) gives finally

$$
R(X, Y) \xi=\kappa(\eta(Y) X-\eta(X) Y)+\mu(\eta(Y) h X-\eta(X) h Y) .
$$

\section{Classification in the 3-dimensional case}

The existence of $(\kappa, \mu, v)$-contact metric manifolds in dimension 3 , as described in Section 4, raises the question of their classification. In this direction, we give some partial answers, assuming additionally that the manifolds satisfy some interesting geometric properties. It is well known that a Riemannian metric $g$ on a compact and orientable manifold $M$ is a critical point of the functional "integral of the scalar curvature $\int_{M} r d v$ " defined on the set of all Riemannian metrics of the same total volume on $M$ if and only if $g$ is an Einstein metric [Besse 1987, page 120]. Now, we consider a 3-dimensional compact contact manifold $(M, \eta)$. Let $\xi$ the characteristic vector field, and let $\mathscr{A}(\eta)$ be the set of Riemannian metrics associated to the contact form $\eta$. Also, it is known that each metric $g \in \mathscr{A}(\eta)$ has the same volume element $d v$. Perrone [1990] showed that a metric $g \in \mathscr{A}(\eta)$ is the critical point of the functional

$$
I(g)=\int_{M} r d v \quad \text { for } g \in \mathscr{A}(\eta)
$$


if and only if

$$
\nabla_{\xi} \tau=0 .
$$

The functional $I$ was studied by Blair and Ledger [1986] in general dimension. Perrone [1990] proved that a contact metric 3-manifold is $\eta$-Einstein if and only if $\sigma=0$, that is, $M$ is $H$-contact and $\nabla_{\xi} \tau=0$. On the other hand, in Blair et al. [1990] proved that a contact metric 3-manifold is $\eta$-Einstein if and only if it is a $(\kappa, 0)$ contact metric manifold. Furthermore, $\eta$-Einstein contact metric 3-manifolds were classified in [Blair and Chen 1992]. Finally, using Theorem 1.1, we deduce

Theorem 5.1. Let $M(\eta, \xi, \phi, g)$ be a complete 3-dimensional $(\kappa, \mu, v)$-contact metric manifold. If $M$ satisfies (5-1), then $M$ is a ( $\kappa, 0)$-contact metric manifold with $\kappa=$ constant. In particular, $M$ is either a Sasakian manifold (if $\kappa=1$ ) or locally isometric to one of the following Lie groups equipped with a left invariant metric: $\mathrm{SU}(2)$ if $0<\kappa<1, \operatorname{SL}(2, \mathbb{R})$ if $\kappa<0$, and $\mathrm{E}(2)$ if $\kappa=0$.

Remark 5.1. Theorem 5.1 could be considered an extension of [Perrone 2006, Theorem 2.3]. In particular, we omit the assumption that $M(\eta, \xi, \phi, g)$ is a homogeneous contact metric 3-manifold.

We now give an example of a compact $(\kappa, 0)$-contact metric manifold $M$ for which the metric $g$ is a critical point of the functional $I$ defined on the set $\mathscr{A}(\eta)$ of the contact manifold $(M, \eta)$. Let $(M, g)$ be a 2-dimensional Riemannian manifold with constant Gaussian curvature $c>-1$. It is known [Blair et al. 1995] that $T_{1} M$ is a $(\kappa, \mu)$-contact metric manifold with $\kappa=c(2-c)$ and $\mu=-2 c$. We apply on $T_{1} M$ a $D$-homothetic transformation with $\alpha=1+c$. Using (3-8), we construct a new contact metric manifold with characteristic vector field belonging to the $(\bar{\kappa}, 0)$-nullity distribution, where $\bar{\kappa}=4 c /(1+c)^{2}<1$.

The relation

$$
\nabla_{\xi} \tau=2 \tau \phi
$$

is the critical point condition of the functional

$$
F(g)=\frac{1}{2} \int_{M}\|\tau\|^{2} d v
$$

defined on the set $\mathscr{A}(\eta)$ of a $(2 n+1)$-dimensional compact contact manifold $(M, \eta)$; see [Chern and Hamilton 1985], [Tanno 1989], and [Blair 2002, p.167]. As an immediate consequence of Theorem 1.1 and [Perrone 2004, Theorem 5.2], we get the following classification of 3-dimensional $(\kappa, \mu, v)$-contact metric manifolds that satisfy (5-2), where the composition $\tau \phi(X, Y)$ has to be interpreted as $\tau(\phi X, Y)$.

Theorem 5.2. Let $M(\eta, \xi, \phi, g)$ be a complete 3-dimensional $(\kappa, \mu, v)$-contact metric manifold for which $\nabla_{\xi} \tau=2 \tau \phi$. Then $M$ is a $(\kappa, 2)$-contact metric manifold 
where $\kappa$ is a constant. If $\kappa=1$ then $M$ is a Sasakian manifold; if $\kappa \neq 1$, it is locally isometric to $\mathrm{SL}(2, \mathbb{R})$ equipped with a left invariant metric. In the second case, any two $(\kappa, 2)$-contact metric manifolds are $D$-invariant under a specific D-homothetic transformation.

Remark 5.2. Let $(M, g)$ be a 2-dimensional Riemannian manifold with constant Gaussian curvature $c=-1$. Then $\left(T_{1} M,\left(\eta, \xi, \phi, g_{S}\right)\right)$ is a $(\kappa, 2)$-contact metric manifold with $\kappa=-3$ [Blair et al. 1995, Theorem 4], where $(\eta, \xi, \phi)$ is the standard contact structure of $T_{1} M$ and $g_{S}$ is the Sasaki metric. The metric $g_{S}$ is a critical point of the functional $F(g)$ defined on the set $\mathscr{A}(\eta)$ of the contact manifold $\left(T_{1} M, \eta\right)$.

Next we consider a 3-dimensional $(\kappa, \mu, v)$-contact metric manifold $M(\eta, \xi, \phi, g)$ with constant $\kappa \neq 1$. Then Equation (4-14) gives $v=0$ everywhere on $M$. Also, using (4-8), (4-11), the main theorem of [Koufogiorgos 1995] and [Blair et al. 1995, Theorem 3], we easily obtain

Theorem 5.3. If $M(\eta, \xi, \phi, g)$ is a 3-dimensional $(\kappa, \mu, v)$-contact metric manifold with constant $\kappa$, then it is a Sasakian manifold when $\kappa=1$ but a $(\kappa, \mu)$-contact metric manifold when $\kappa<1$. In the second case, $M$ is locally isometric to one of the following Lie groups with a left invariant metric: $\mathrm{SU}(2) ; \mathrm{SL}(2, \mathbb{R}) ; \mathrm{E}(2)$, the group of rigid motions of Euclidean 2-space; or E(1,1), the group of rigid motions of Minkowski 2-space.

Remark 5.3. Examples 4.2 and 4.3 show that the condition that $v$ is constant does not imply that the other functions $\kappa$ and $\mu$ are constant. The same applies, of course, with $\mu$ and $v$ interchanged.

Using Theorem 1.1, we can reformulate some known results in terms of $(\kappa, \mu, v)$ contact metric manifolds. For example, [Calvaruso et al. 1999, Theorem 4.1] (using also [Tanno 1967] in the Sasakian case) and [Perrone 2002, Corollary 3.2].

Remark 5.4. In Examples 4.1, 4.2, and 4.3, the eigenvalues of $h$ are nonconstant smooth functions. This shows that $(\kappa, \mu, v)$-contact metric manifolds are not necessarily locally homogeneous contact Riemannian manifolds [Perrone 1998] or, more generally, curvature homogeneous. However, $(\kappa, \mu)$-contact metric manifolds are always locally homogeneous contact manifolds; see [Boeckx 2000]. For more details about homogeneous Riemannian manifolds, see [Tricerri and Vanhecke 1983].

Acknowledgments. Markellos acknowledges Assistant Professor Andreas Arvanitoyeorgos for several useful comments on the manuscript. The authors thank the referee for useful comments on the manuscript, especially on Section 5. 


\section{References}

[Besse 1987] A. L. Besse, Einstein manifolds, vol. 10, Ergebnisse der Mathematik, Springer, Berlin, 1987. MR 88f:53087 Zbl 0613.53001

[Blair 1976] D. E. Blair, Contact manifolds in Riemannian geometry, Springer, Berlin, 1976. Lecture Notes in Mathematics, Vol. 509. MR 57 \#7444 Zbl 0319.53026

[Blair 2002] D. E. Blair, Riemannian geometry of contact and symplectic manifolds, Progress in Mathematics 203, Birkhäuser, Boston, 2002. MR 2002m:53120 Zbl 1011.53001

[Blair and Chen 1992] D. E. Blair and H. Chen, "A classification of 3-dimensional contact metric manifolds with $Q \phi=\phi Q$. II”, Bull. Inst. Math. Acad. Sinica 20:4 (1992), 379-383. MR 94b:53062 Zbl 0767.53023

[Blair and Ledger 1986] D. E. Blair and A. J. Ledger, "Critical associated metrics on contact manifolds. II”, J. Austral. Math. Soc. Ser. A 41:3 (1986), 404-410. MR 87k:58060 Zbl 0611.53031

[Blair et al. 1990] D. E. Blair, T. Koufogiorgos, and R. Sharma, "A classification of 3-dimensional contact metric manifolds with $Q \phi=\phi Q "$, Kodai Math. J. 13:3 (1990), 391-401. MR 91j:53015 Zbl 0716.53041

[Blair et al. 1995] D. E. Blair, T. Koufogiorgos, and B. J. Papantoniou, "Contact metric manifolds satisfying a nullity condition”, Israel J. Math. 91:1-3 (1995), 189-214. MR 96f:53037 Zbl 0837.53038

[Boeckx 2000] E. Boeckx, "A full classification of contact metric $(k, \mu)$-spaces", Illinois J. Math. 44:1 (2000), 212-219. MR 2001b:53099 Zbl 0969.53019

[Calvaruso et al. 1999] G. Calvaruso, D. Perrone, L. Vanhecke, "Homogeneity on three-dimensional contact metric manifolds”, Israel J. Math. 114 (1999), 301-321. MR 2000j:53105 Zbl 0957.53017

[Chern and Hamilton 1985] S. S. Chern and R. S. Hamilton, "On Riemannian metrics adapted to three-dimensional contact manifolds", pp. 279-308 in Workshop Bonn 1984, edited by F. Hirzebruch et al., Lecture Notes in Math. 1111, Springer, Berlin, 1985. MR 87b:53060 Zbl 0561.53039

[Gil-Medrano 2001] O. Gil-Medrano, "Relationship between volume and energy of vector fields", Differential Geom. Appl. 15:2 (2001), 137-152. MR 2002f:53112 Zbl 1066.53068

[González-Dávila and Vanhecke 2001] J. C. González-Dávila and L. Vanhecke, "Minimal and harmonic characteristic vector fields on three-dimensional contact metric manifolds", J. Geom. 72:1-2 (2001), 65-76. MR 2003a:53113 Zbl 1005.53039

[Han and Yim 1998] D.-S. Han and J.-W. Yim, "Unit vector fields on spheres, which are harmonic maps”, Math. Z. 227:1 (1998), 83-92. MR 99c:58044 Zbl 0891.53024

[Koufogiorgos 1995] T. Koufogiorgos, "On a class of contact Riemannian 3-manifolds", Results Math. 27:1-2 (1995), 51-62. MR 95m:53040 Zbl 0833.53032

[Koufogiorgos and Tsichlias 2000] T. Koufogiorgos and C. Tsichlias, "On the existence of a new class of contact metric manifolds", Canad. Math. Bull. 43:4 (2000), 440-447. MR 2001h:53113 Zbl 0978.53086

[O'Neill 1983] B. O'Neill, Semi-Riemannian geometry. With applications to relativity, Pure and Applied Mathematics 103, Academic Press, New York, 1983. MR 85f:53002 Zbl 0531.53051

[Perrone 1990] D. Perrone, "Torsion and critical metrics on contact three-manifolds", Kodai Math. J. 13:1 (1990), 88-100. MR 91b:58038 Zbl 0709.53034

[Perrone 1996] D. Perrone, "Ricci tensor and spectral rigidity of contact Riemannian 3-manifolds", Bull. Inst. Math. Acad. Sinica 24:2 (1996), 127-138. MR 97e:53092 Zbl 0859.53028

[Perrone 1998] D. Perrone, "Homogeneous contact Riemannian three-manifolds", Illinois J. Math. 42:2 (1998), 243-256. MR 99a:53067 Zbl 0906.53031 
[Perrone 2002] D. Perrone, "Weakly $\phi$-symmetric contact metric spaces", Balkan J. Geom. Appl. 7:2 (2002), 67-77. MR 2003k:53103 Zbl 1028.53047

[Perrone 2003] D. Perrone, "Harmonic characteristic vector fields on contact metric three-manifolds”, Bull. Austral. Math. Soc. 67:2 (2003), 305-315. MR 2004c:53097 Zbl 1034.53050

[Perrone 2004] D. Perrone, "Contact metric manifolds whose characteristic vector field is a harmonic vector field”, Differential Geom. Appl. 20:3 (2004), 367-378. MR 2005a:53134 Zbl 1061. 53028

[Perrone 2006] D. Perrone, "Taut contact circles on H-contact 3-manifolds", Int. Math. Forum 1:2528 (2006), 1285-1296. MR 2007f:53096 Zbl 05151892

[Tanno 1967] S. Tanno, "Locally symmetric $K$-contact Riemannian manifolds", Proc. Japan Acad. 43 (1967), 581-583. MR 37 \#861 Zbl 0155.49802

[Tanno 1968] S. Tanno, "The topology of contact Riemannian manifolds", Illinois J. Math. 12 (1968), 700-717. MR 38 \#2803 Zbl 0165.24703

[Tanno 1989] S. Tanno, "Variational problems on contact Riemannian manifolds", Trans. Amer. Math. Soc. 314:1 (1989), 349-379. MR 90f:53071 Zbl 0677.53043

[Tricerri and Vanhecke 1983] F. Tricerri and L. Vanhecke, Homogeneous structures on Riemannian manifolds, London Mathematical Society Lecture Note Series 83, Cambridge University Press, Cambridge, 1983. MR 85b:53052 Zbl 0509.53043

[Wiegmink 1995] G. Wiegmink, "Total bending of vector fields on Riemannian manifolds", Math. Ann. 303:2 (1995), 325-344. MR 97a:53050 Zbl 0834.53034

[Wood 1997] C. M. Wood, "On the energy of a unit vector field", Geom. Dedicata 64:3 (1997), 319-330. MR 98e:58064 Zbl 0878.58017

Received April 18, 2007. Revised October 10, 2007.

\author{
THEMIS Koufogiorgos \\ UNIVERSITY OF IOANNINA \\ DEPARTMENT OF MATHEMATICS \\ 45100 IOANNINA \\ GREECE \\ tkoufog@cc.uoi.gr \\ Michael Markellos \\ UNIVERSITY OF PATRAS \\ DEPARTMENT OF MATHEMATICS \\ 26500 RION \\ GREECE \\ mark@upatras.gr

VASSILIS J. PAPANTONIOU
UNIVERSITY OF PATRAS
DEPARTMENT OF MATHEMATICS
26500 RION
GREECE

bipapant@math.upatras.gr 